\title{
Sulfated and Phosphated Mesoporous Nb Oxide in the Benzylation of Anisole and Toluene by Benzyl Alcohol \\ (Supporting Information)
}

Yuxiang Rao, ${ }^{\mathrm{a}}$ Michel Trudeau, ${ }^{\mathrm{b}}$ and David Antonelli ${ }^{\mathrm{a}}$

${ }^{a}$ Department of Chemistry and Biochemistry, University of Windsor, 401 Sunset Avenue, Windsor, Ontario N9B 3P4, Canada, Fax: +1(519) 973-7098

${ }^{\mathrm{b}}$ M. L. Trudeau, Emerging Technologies, Hydro-Québec Research Institute, 1800 Boul. Lionel-Boulet, Varennes, Quebec, Canada J3X 1S1

Correspondence to David Antonelli, ${ }^{a}$ Department of Chemistry and Biochemistry, University of Windsor, 401 Sunset Avenue, Windsor, Ontario N9B 3P4, Canada, Fax: +1(519) 973-7098

E-mail: danton@uwindsor.ca

\section{Synthesis of mesoporous niobium oxide materials}

In a typical preparation, niobium ethoxide $(50 \mathrm{~g}, 157.1 \mathrm{mmol})$ was warmed with dodecylamine $(8.7372 \mathrm{~g}$, 47.13mmol) using a heat gun until a homogeneous colorless solution was obtained. To this solution was added 1L of distilled water with stirring. A white gelatinous precipitates formed immediately. After precipitation occurred, hydrochloric acid $(0.1743 \mathrm{~g}, 4.713 \mathrm{mmol})$ was added directly to the solution before the white solid was allowed to sit at room temperature overnight. The mixture was heated at $40^{\circ} \mathrm{C}$ for 2 days, $60^{\circ} \mathrm{C}$ for 2 days, $80^{\circ} \mathrm{C}$ for 2 days, and $95^{\circ} \mathrm{C}$ for 4 days. After filtration, the solid was dried in an oven at $95^{\circ} \mathrm{C}$ for $1 \sim 2$ hours, sealed in a tube, and heated to $120^{\circ} \mathrm{C}$ for 2 days and $140^{\circ} \mathrm{C}$ for 2 days. The product was then collected and washed five times with methanol to remove the surfactant. Each washing cycle was conducted for $24 \mathrm{~h}$ in a large beaker with vigorous stirring followed by filtration. For the first wash, p-toluene sulfonic acid $(9.8615 \mathrm{~g}$, $51.843 \mathrm{mmol}$ ) was added to the solution with $200 \mathrm{ml}$ diethyl ether. For the second wash, $0.8965 \mathrm{~g}$ p-toluene sulfonic acid was added to methanol solution. The solid was then collected and dried for $24 \mathrm{~h}$ at $120^{\circ} \mathrm{C}$. The template-free samples were then treated with $1 \mathrm{M}$ sulfuric acid or phosphoric acid (in methanol) and filtered to obtain the mesoporous sulfated or phosphated $\mathrm{Nb}$ oxides. 


\section{Characterization}

X-ray powder diffraction data were recorded on a Siemens D5000-20 diffractometer using $\mathrm{Cu} \mathrm{Ka}$ radiation. Nitrogen adsoption and desorption data were collected on a Micromeritics ASAP 2010. High resolution transmission electron microscopy (TEM) images were obtained by using a H9000 HR-TEM operated at $300 \mathrm{kV}$. FTIR experiments were performed on Bruker Vector 22 FT-IR spectrometer. Elemental analyses were performed by Galbraith lab (Knoxville, TN) for P and S, and locally at the University of Windsor for C, H, and N on a Perkin Elmer Series II Analyzer 2400.

\section{Catalytic Runs}

A $100 \mathrm{~mL}$ round-bottom 3-neck flask provided with a reflux condenser was used as a stirred bed reactor to test the catalytic activities of the materials. Nitrogen was introduced into the flask through one of the gas inlets. The second inlet was equipped with a septum for sample removal. $50 \mathrm{ml}$ of Anisole or Toluene and $5 \mathrm{ml}$ of Benzyl alcohol were added to the reactor with $0.5 \mathrm{~g}$ catalyst at each run. The reactant mixture was refluxed with stirring at atmospheric pressure and constant temperature (controlled oil bath temperatures of $170^{\circ} \mathrm{C}$ for anisole and $130^{\circ} \mathrm{C}$ for toluene). Samples of the reaction mixture were periodically withdrawn and analyzed by using a Varian CP-3800 gas chromatograph equipped with a hydrogen flame ionization detector system and capillary column CP-SIL 5CB (15 m, $0.25 \mathrm{~mm}$ ID), the temperature was programmed from 50 to $300{ }^{\circ} \mathrm{C}$ $\left(10^{\circ} \mathrm{C} / \mathrm{min}\right)$ with $\mathrm{H}_{2} 1 \mathrm{ml} / \mathrm{min}$ as carrier gas. The results from $\mathrm{GC}$ identify the o- and p- benzylation products and benzyl ether as the main products of these reactions. Activities were calculated on the basis of percentage conversion benzyl alcohol in the starting mixture. 
Table S1. Distribution of the acidic strength with Hammett indicators

\begin{tabular}{|c|c|c|c|c|c|c|c|}
\hline Indicator & pKa & $\mathrm{Nb}_{2} \mathrm{O}_{5}$ & $\begin{array}{c}\mathrm{H}_{2} \mathrm{SO} 4 \\
/ \mathrm{Nb}_{2} \mathrm{O}_{5} \\
\end{array}$ & $\begin{array}{c}\mathrm{H}_{3} \mathrm{PO}_{4} \\
/ \mathrm{Nb}_{2} \mathrm{O}_{5} \\
\end{array}$ & $\begin{array}{l}\text { Meso } \\
\mathrm{Nb} \\
\end{array}$ & $\begin{array}{l}\mathrm{H}_{2} \mathrm{SO}_{4} \\
/ \mathrm{Meso} \mathrm{Nb}\end{array}$ & $\begin{array}{l}\mathrm{H}_{3} \mathrm{PO}_{4} \\
/ \text { Meso } \mathrm{Nb}\end{array}$ \\
\hline Methyl red & +5.0 & + & + & + & + & + & + \\
\hline Methyl yellow & +3.3 & + & + & + & + & + & + \\
\hline Crystal Violet & +0.8 & - & + & + & + & + & + \\
\hline Dicinnamalacetone & -3.0 & - & + & + & + & + & + \\
\hline 2,4-Dinitroaniline & -4.4 & - & - & - & + & + & + \\
\hline Benzalacetophenone & -5.6 & - & - & - & + & + & + \\
\hline 2-Bromo-4,6-dinitroaniline & -6.6 & - & - & - & + & + & + \\
\hline 9,10-Anthraquinone & -8.2 & - & - & - & - & + & + \\
\hline 3-Nitrotoluene & -11.99 & - & - & - & - & - & - \\
\hline 1-chloro-4-Nitrobenzene & -12.70 & - & - & - & - & - & - \\
\hline 2,4-Dinitrofluorobenzene & -14.52 & - & - & - & - & - & - \\
\hline
\end{tabular}

$$
\text { “+”: color changed “-": color unchanged }
$$

Table S2. The amount of acids as mmole g-1 which was calculated from the n-butylamine titration

\begin{tabular}{|c|c|c|c|c|c|c|}
\hline Indicator & $\mathbf{N b}_{2} \mathbf{O}_{\mathbf{5}}$ & $\begin{array}{c}\mathbf{H}_{2} \mathbf{S O}_{4} / \\
\mathbf{N b}_{2} \mathbf{O}_{\mathbf{5}}\end{array}$ & $\begin{array}{c}\mathbf{H}_{3} \mathbf{P O}_{4} / \\
\mathbf{N b}_{2} \mathbf{O}_{5}\end{array}$ & Meso $\mathbf{~ b b}$ & $\begin{array}{c}\mathbf{H}_{2} \mathbf{S O}_{4} / \\
\text { Meso } \mathbf{~ N b}\end{array}$ & $\begin{array}{c}\mathbf{H}_{3} \mathbf{P O}_{4} / \\
\text { Meso } \mathbf{~ N b}\end{array}$ \\
\hline Methyl yellow & 0.024 & 0.338 & 0.317 & 2.478 & 31.784 & 3.086 \\
\hline
\end{tabular}

Table S3.The internal structure and surface properties of catalysts before and after reactions

\begin{tabular}{|c|c|c|c|}
\hline Sample & $\begin{array}{c}\text { BET surface area } \\
\left(\mathrm{m}^{2} / \mathrm{g}\right)\end{array}$ & $\begin{array}{l}\text { Volume } \\
\left(\mathrm{cm}^{3} / \mathrm{g}\right) \\
\end{array}$ & $\begin{array}{c}\text { BJH Pore size } \\
\text { (A) }\end{array}$ \\
\hline $\mathrm{Nb}_{2} \mathrm{O}_{5}$ & 3.63 & N/A & N/A \\
\hline $\mathrm{H}_{2} \mathrm{SO}_{4} / \mathrm{Nb}_{2} \mathrm{O}_{5}$ & 5.29 & N/A & N/A \\
\hline $\mathrm{H}_{3} \mathrm{PO}_{4} / \mathrm{Nb}_{2} \mathrm{O}_{5}$ & 2.96 & N/A & N/A \\
\hline Meso $\mathrm{Nb}$ & 612.63 & 0.3956 & 22.7 \\
\hline *Meso Nb (Anisole) & 587.90 & 0.3123 & 20.6 \\
\hline *Meso Nb (Toluene) & 386.04 & 0.2088 & 20.6 \\
\hline $\mathrm{H}_{2} \mathrm{SO} 4 / \mathrm{Meso} \mathrm{Nb}$ & 519.10 & 0.3408 & 20.6 \\
\hline${ }^{*} \mathrm{H}_{2} \mathrm{SO} 4 / \mathrm{Meso} \mathrm{Nb}$ (Anisole) & 84.71 & 0.1409 & 37.2 \\
\hline${ }^{*} \mathrm{H}_{2} \mathrm{SO} 4 / \mathrm{Meso} \mathrm{Nb}$ (Toluene) & 3.35 & 0.004 & 51.1 \\
\hline $\mathrm{H}_{3} \mathrm{PO} 4 / \mathrm{Meso} \mathrm{Nb}$ & 502.81 & 0.3286 & 20.7 \\
\hline${ }^{*} \mathrm{H}_{3} \mathrm{PO} 4 / \mathrm{Meso} \mathrm{Nb}$ (Anisole) & 76.38 & 0.0932 & 39.5 \\
\hline${ }^{*} \mathrm{H}_{3} \mathrm{PO} 4 / \mathrm{Meso} \mathrm{Nb}$ (Toluene) & 6.41 & 0.0146 & 47.0 \\
\hline
\end{tabular}

* Denotes surface areas after reaction with substrate in bracket. 

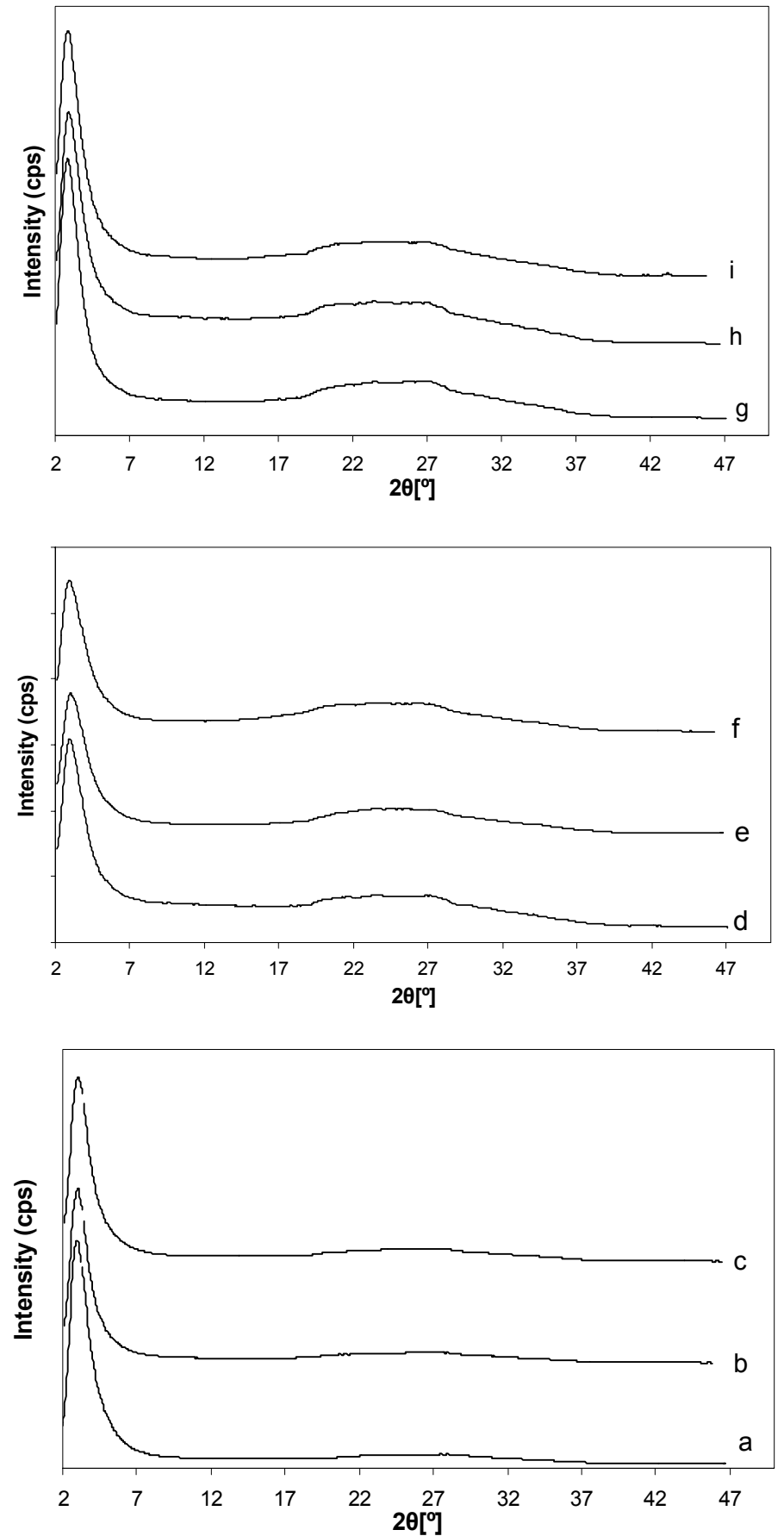

Figure S1 X-ray diffraction patterns of Nb-TMS samples. a) meso $\mathrm{Nb}$ oxide, b) meso $\mathrm{Nb}$ oxide after reaction with with anisole, c) meso $\mathrm{Nb}$ oxide after reaction with toluene, d) $\mathrm{H}_{2} \mathrm{SO}_{4} /$ meso $\mathrm{Nb}$ oxide, e) $\mathrm{H}_{2} \mathrm{SO}_{4} / \mathrm{meso}^{\mathrm{Nb}}$ oxide after reaction with anisole, f) $\mathrm{H}_{2} \mathrm{SO}_{4} /$ meso $\mathrm{Nb}$ oxide after reaction with toluene, g) $\mathrm{H}_{3} \mathrm{PO}_{4} /$ meso $\mathrm{Nb}$ oxide, h) $\mathrm{H}_{3} \mathrm{PO}_{4} / \mathrm{meso}$ $\mathrm{Nb}$ oxide after reaction with anisole, i) $\mathrm{H}_{3} \mathrm{PO}_{4} /$ meso $\mathrm{Nb}$ oxide after reaction with toluene 


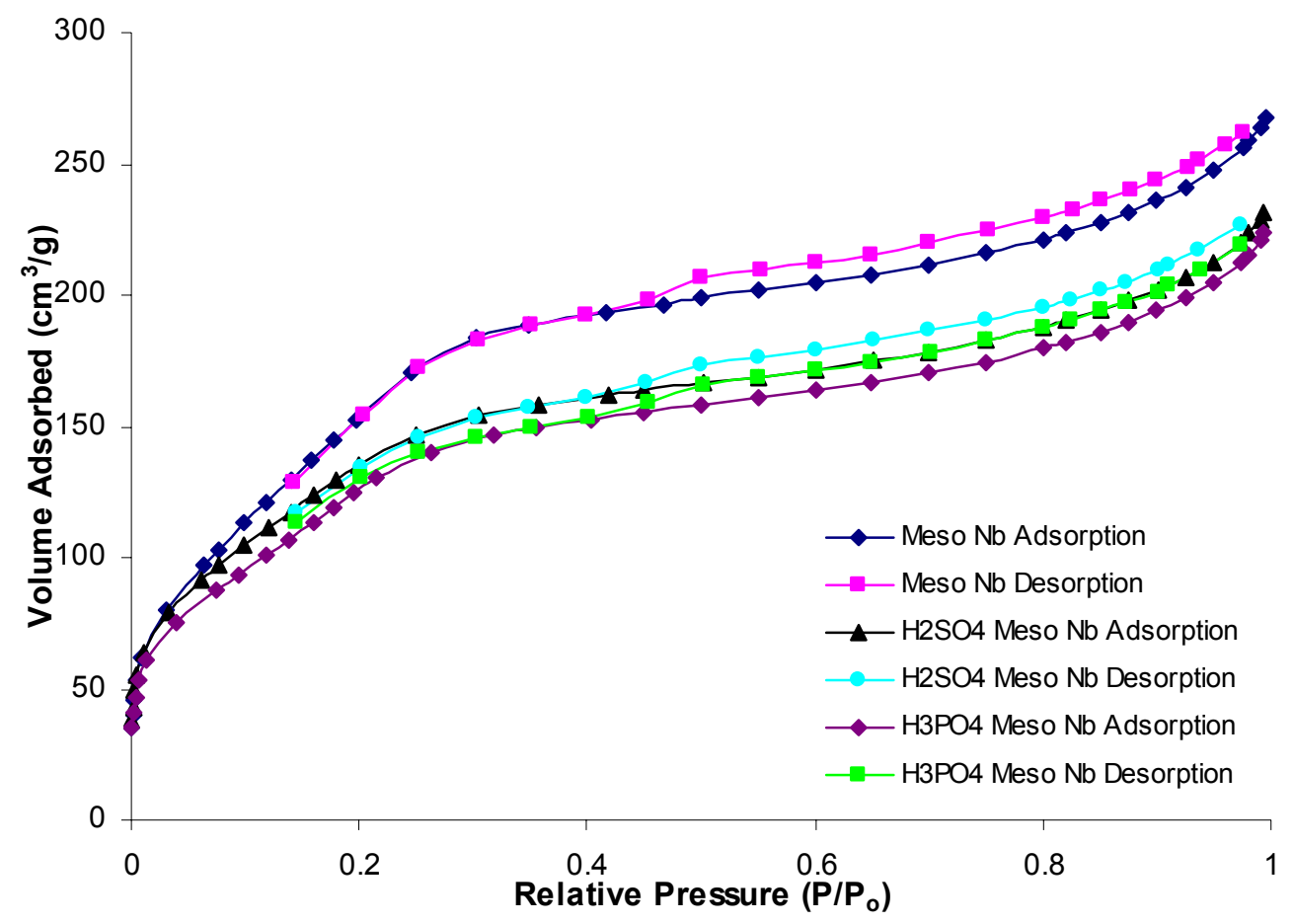

Figure S2. Nitrogen adsorption/desorption isotherms of different microporous $\mathrm{Nb}$ oxides synthesized with n-dodecylamine.

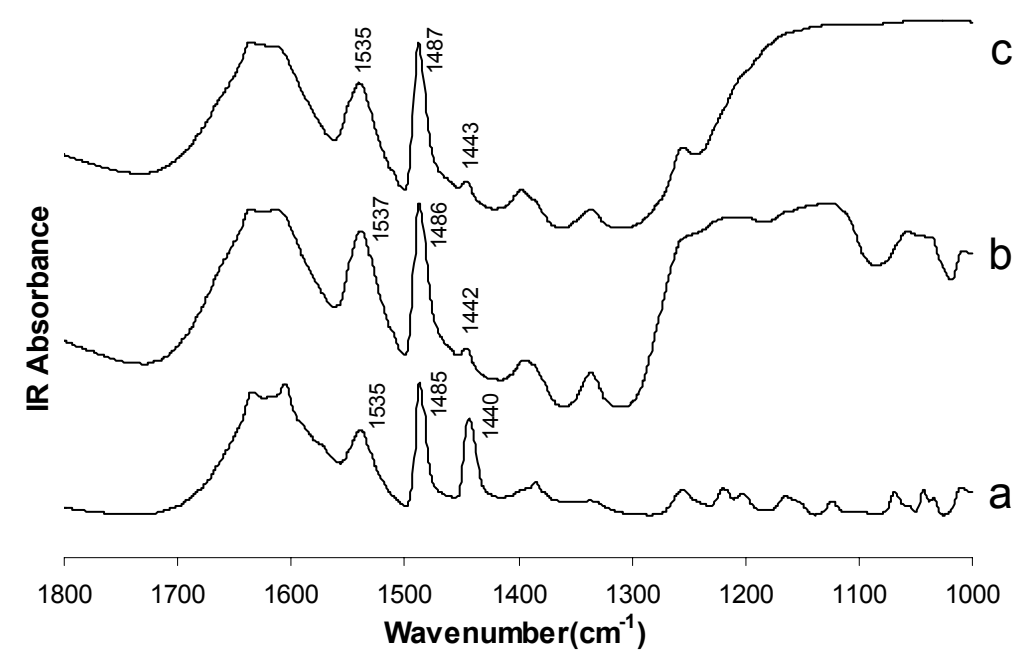

Figure S3. The FTIR spectra of different meso $\mathrm{Nb}$ oxides after pyridine vapor adsorption. a) Meso $\mathrm{Nb}, \mathrm{b}$ )

$$
\left.\mathrm{H}_{2} \mathrm{SO}_{4} / \mathrm{Meso} \mathrm{Nb}, \mathrm{c}\right) \mathrm{H}_{3} \mathrm{PO}_{4} / \mathrm{Meso} \mathrm{Nb}
$$




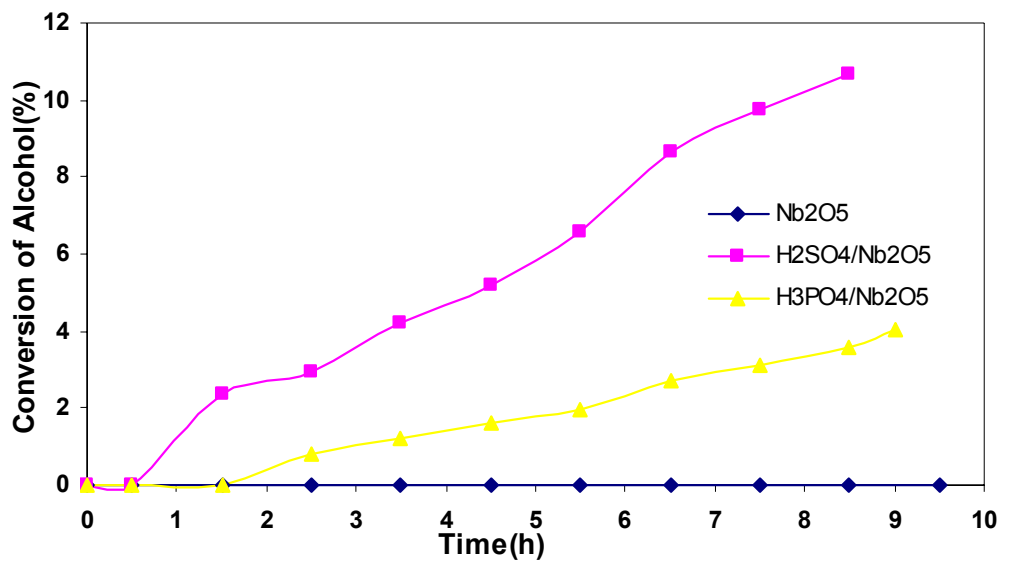

Figure S4 Percent conversion of benzyl alcohol in the benzylation of anisole catalyzed by different bulk Nb oxides

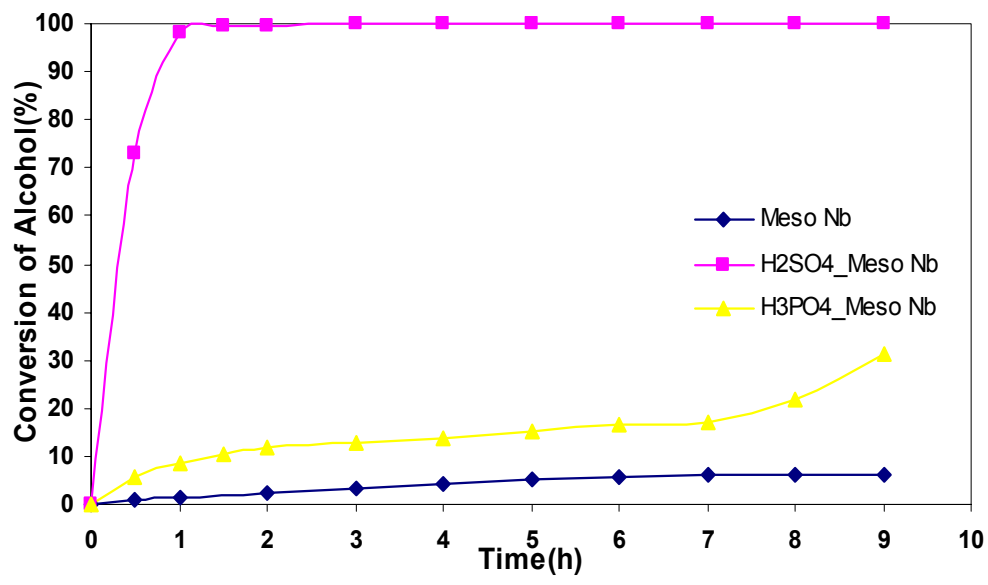

Figure S5 Percent conversion of benzyl alcohol in the benzylation of toluene catalyzed by different mesoporous $\mathrm{Nb}$ oxides 